\title{
A DELAYED MATHEMATICAL MODEL FOR TESTOSTERONE SECRETION WITH FEEDBACK CONTROL MECHANISM
}

\author{
BANIBRATA MUKHOPADHYAY and RAKHI BHATTACHARYYA
}

Received 7 July 2003 and in revised form 21 July 2003

\begin{abstract}
A mathematical model describing the biochemical interactions of the luteinizing hormone (LH), luteinizing hormone-releasing hormone (LHRH), and testosterone (T) is presented. The model structure consists of a negative feedback mechanism with transportation and secretion delays of different hormones. A comparison of stability and bifurcation analysis in the presence and absence of delays has been performed. Mathematical implications of castration and testosterone infusion are also studied.
\end{abstract}

2000 Mathematics Subject Classification: 37G15.

1. Introduction. In humans and many other animals, the hormone testosterone (T) is considered to be an extremely important hormone. Any regular imbalance of this hormone may cause dramatic behavioral changes. Men have a T level of between 10-35 nanomoles per litre of blood. Reduced levels of $\mathrm{T}$ are often accompanied by personality changes - the individual tends to become less forceful and commanding. Increased levels of $\mathrm{T}$, on the other hand, induce the converse. In men, $\mathrm{T}$ is primarily generated from the interstitial cells of the testis, which produces about ninety percent with the rest coming from other parts of the endocrine system. Though the physiological process of generation is not yet fully understood, there is general agreement on certain key elements. The luteinizing hormone-releasing hormone (LHRH) is normally secreted by the hypothalamus and is carried to the pituitary in the blood. The anterior pituitary, under the influence of LHRH, secretes the luteinizing hormone (LH). LH, in turn, stimulates the interstitial cells of the testis and generates the hormone T. This hormone is thought to have a feedback effect on the secretion of its precursor hormones LH and LHRH.

There are several reviews of the experimental data regarding the validity of the LHRHLH-T system [1, 3, 4, 6]. An important recurrent observation in many experiments on intact adult animals is that the serum concentration of both LH and T undergo rapid cyclic fluctuations of roughly the same period in each individual, but varying slightly between individuals. This is called the phenomenon of pulsatile or episodic hormone release. Katongole et al. [9] measured LH and T concentrations in the peripheral plasma of bulls and observed cyclic fluctuations of varying periods from animal to animal ranging from 2 to 5 hours. They concluded that the cyclic pattern of LH release is due to some inherent rhythm, and each transient LH peak results in transient maximal stimulation of testicular T secretion. Nankin and Troen [12] measured LH concentration in men and found regular cyclic periods of 1-3 hours. 
Experiments in which the natural state of the animals has been disturbed have also been conducted. Bolt [2] found that the cyclic fluctuations of LH in rams could be supported by infusion of T. Moger and Armstrong [10] observed elevations of T concentrations in rats following acute LH treatment and found differences between immature and mature animals. Pelletier [13] observed the elevation of LH levels in rams following castration and found that subsequent injection of T caused these levels to decrease [14].

The purpose of the present paper is concerned primarily with analyzing the phenomenon of periodic fluctuations in serum concentration of the hormone testosterone referred to in the literature as pulsatile or episodic release. This is accomplished by establishing the existence of periodic solutions of systems of autonomous differential equations which describe biochemical systems exhibiting negative feedback. Next, we introduce delays in the system arising from the time taken by the hormones to travel from source to destination through blood circulation and the time required for the synthesis of $\mathrm{T}$ and compare the oscillatory behaviour of the system with and without delays. Finally, we consider the biological implications of castration and T infusion into the system.

2. Description of the model. In the present study, a mathematical model concerning the LHRH-LH-T system is considered. There are three active components of the system - the hypothalamus, the anterior pituitary, and the testis. The hypothalamus secretes LHRH into the hypophyseal portal vessels which causes pituitary to secrete LH into the general circulation which in turn causes the gonadal secretion of T. This hormone has a negative feedback effect on the hypothalamic LHRH secretion rate. The long feedback loop from testis to hypothalamus is considered. The exclusion of the short feedback loop from gonads to the pituitary is supported by experimental evidences. We also exclude the short pituitary to hypothalamus loop for simplicity. Experimental results obtained by Carmel et al. [5] suggest the existence of a neural clock, a pulse generator which forces the hypothalamic secretion of LHRH and thus driving the entire system. In this paper, we neglect these neural input terms and consider only the autonomous LHRH-LH-T system for the well-known fact that the autonomous system may still principally govern the total system behaviour with the forcing terms only produce small perturbations.

The rate of LH release from the pituitary is assumed to depend linearly on the local concentration of LHRH, and the rate of T synthesis by the testis depends linearly on the local LH concentration. The synthesis of $\mathrm{T}$ takes a fixed finite time. Thus there is a time delay between the stimulation of the interstitial cells of the testis by LH and the eventual release of $\mathrm{T}$ into the blood stream. There are also transport delays owing to the time taken for the hormones to travel from source to destination across the body due to the spatial separation of different components of the model. Each of the hormones is cleared from the bloodstream at a rate proportional to its concentration, that is, according to first-order kinetics. Thus the concentration of each hormone is affected by the complementary process of synthesis and clearance. We have not also included, in our model, sources of T other than the gonads. The adrenals, for example, are an important extragonadal source of this hormone. In adult animals, however, the principal source is the testis and we thus consider this source only in our model. 
We give a mathematical realization of all these considerations into the following model:

$$
\begin{aligned}
& \frac{d R}{d t}=f(T)-d_{1} R(t), \\
& \frac{d L}{d t}=r_{2} R\left(t-\tau_{1}\right)-d_{2} L(t), \\
& \frac{d T}{d t}=r_{3} L\left(t-\tau_{0}-\tau_{2}\right)-d_{3} T(t),
\end{aligned}
$$

where $R(t)$ is the concentration of LHRH at the hypothalamus at time $t, L(t)$ is the concentration of LH at the pituitary at time $t, T(t)$ is the serum concentration of T in the vicinity of testis at time $t$, and $d_{1}, d_{2}$, and $d_{3}$ are the decay rates of the three hormones in the bloodstream. Moreover, $r_{2}$ and $r_{3}$ are the response rates for the production of $\mathrm{LH}$ and $\mathrm{T}, \tau_{1}$ and $\tau_{2}$ are the time taken by the hormones to be transported between the hypothalamus and pituitary $\left(\tau_{1}\right)$ and between the pituitary and the testis $\left(\tau_{2}\right), \tau_{0}$ is the time interval between testis stimulation and T secretion, and $f(T)$ is the feedback function which is positive monotone decreasing in nature.

THEOREM 2.1. All the solutions of system (2.1) are bounded.

Proof. From system (2.1),

$$
\frac{d}{d t}(R+L+T)=f(T)-d_{1} R+r_{2} R-d_{2} L+r_{3} L-d_{3} T \leq f(T)+r_{2} R+r_{3} L
$$

Since $f(T)$ is positive monotone decreasing, there exists a positive number $F$ such that $f(T) \leq F$. Therefore,

$$
\frac{d}{d t}(R+L+T) \leq F+r_{2} R+r_{3} L \leq F+r(R+L)
$$

where $r=\min \left(r_{2}, r_{3}\right)$. Hence the theorem follows.

The biological significance of Theorem 2.1 is that the blood concentration of different hormones in an individual cannot exceed a certain limit which ensures the biological validity of the model considered.

The only interior equilibrium point $E^{*}\left(R^{*}, L^{*}, T^{*}\right)$ of the system is given by

$$
L^{*}=\frac{d_{3} T^{*}}{r_{3}}, \quad R^{*}=\frac{d_{2} d_{3} T^{*}}{r_{2} r_{3}}, \quad f\left(T^{*}\right)-\frac{d_{1} d_{2} d_{3} T^{*}}{r_{2} r_{3}}=0 .
$$

3. Stability and bifurcation analysis in the absence of delays. In Section 2, we have considered an LHRH-LH-T model given by (2.1). We now study the stability of the steady state and its bifurcation behaviour. We linearise the model with the following transformations:

$$
x=R-R^{*}, \quad y=L-L^{*}, \quad z=T-T^{*} .
$$


The linearised model is

$$
\begin{aligned}
& \frac{d x}{d t}=f^{\prime}\left(T^{*}\right) z(t)-d_{1} x(t), \\
& \frac{d y}{d t}=r_{2} x\left(t-\tau_{1}\right)-d_{2} y(t), \\
& \frac{d z}{d t}=r_{3} y\left(t-\tau_{2}-\tau_{0}\right)-d_{3} z(t) .
\end{aligned}
$$

We look for solutions of (3.2) in the form

$$
x=\alpha_{1} e^{\lambda t}, \quad y=\alpha_{2} e^{\lambda t}, \quad z=\alpha_{3} e^{\lambda t}
$$

The corresponding characteristic equation will be given by

$$
\lambda^{3}+A_{1} \lambda^{2}+A_{2} \lambda+A_{3}+A_{4} e^{-\lambda\left(\tau_{0}+\tau_{1}+\tau_{2}\right)}=0
$$

where

$$
\begin{aligned}
A_{1}=d_{1}+d_{2}+d_{3}, & A_{2}=d_{1} d_{2}+d_{2} d_{3}+d_{3} d_{1}, \\
A_{3}=d_{1} d_{2} d_{3}, & A_{4}=-f^{\prime}\left(T^{*}\right) r_{2} r_{3} .
\end{aligned}
$$

In the absence of delays, (3.4) becomes

$$
\lambda^{3}+A_{1} \lambda^{2}+A_{2} \lambda+A_{3}+A_{4}=0
$$

THEOREM 3.1. In absence of delays, system (2.1) will be unstable if

$$
\frac{A_{1} A_{2}}{A_{3}} \geq 9
$$

Proof. The condition of unstability of system (2.1) is

$$
A_{1} A_{2}-A_{3}-A_{4}<0
$$

as $A_{1}$ and $A_{3}+A_{4}$ are both positive. Applying the following inequality of Hardy et al. [8]:

$$
\frac{1}{3}\left(d_{1}+d_{2}+d_{3}\right) \geq \frac{1}{3}\left[d_{1} d_{2}+d_{2} d_{3}+d_{3} d_{1}\right]^{1 / 2} \geq\left(d_{1} d_{2} d_{3}\right)^{1 / 3}
$$

for $d_{1}, d_{2}, d_{3}>0$ (equality sign holds only for $d_{1}=d_{2}=d_{3}$ ), we get

$$
\frac{A_{1} A_{2}}{A_{3}}-1 \geq 8
$$

that is, $A_{1} A_{2} / A_{3} \geq 9$ which completes the proof. 
We consider the feedback function as (see [11])

$$
f(T)=\frac{A}{k+T^{m}},
$$

where $A$ and $k$ are positive constants, and $m$ is a positive number known as Hill coefficient. From the model equation and from the feedback function $f(T)$, it follows that, for small $k$, the rate of production of LHRH will be high.

Using the form of $f(T)$ as mentioned above, we get from Theorem 3.1 that the condition for unstability of the system, for small $k$, is $m>8$.

THEOREM 3.2. In absence of delays, system (2.1) with $f(T)$ as in (3.11) will undergo a Hopf bifurcation at the parametric value $k=k_{c}$.

Proof. Let $\lambda=a+i b$ be a root of (3.6). Putting the value of $\lambda$ in (3.6) and separating real and imaginary parts, we get

$$
\begin{gathered}
a^{3}-3 a b^{2}+A_{1} a^{2}-A_{1} b^{2}+A_{2} a+A_{3}+A_{4}=0, \\
3 a^{2} b-b^{3}+A_{1} a b+A_{2} b=0 .
\end{gathered}
$$

The condition for change of stability of $E^{*}$ is that (3.6) has a purely imaginary solution, that is,

$$
\lambda=i b
$$

which implies $a=0$. Using this value of $a$ in (3.12) and (3.13), we get

$$
\begin{gathered}
-A_{1} b^{2}+A_{3}+A_{4}=0, \\
-b^{3}+A_{2} b=0 .
\end{gathered}
$$

Equations (3.15) and (3.16) together give

$$
A_{1} A_{2}=A_{3}+A_{4}
$$

Using (2.4), (3.5), and (3.11), we get from (3.17)

$$
A_{1} A_{2}-d_{1} d_{2} d_{3}=\frac{d_{1} d_{2} d_{3} m\left(T^{*}\right)^{m}}{k+\left(T^{*}\right)^{m}}
$$

which gives the value of " $k$ " as

$$
\begin{aligned}
k & =\frac{A_{1} A_{2}\left(T^{*}\right)^{m}-d_{1} d_{2} d_{3}(m+1)\left(T^{*}\right)^{m}}{d_{1} d_{2} d_{3}-A_{1} A_{2}} \\
& =\frac{A_{1} A_{2}\left(T^{*}\right)^{m}-A_{3}(m+1)\left(T^{*}\right)^{m}}{A_{3}-A_{1} A_{2}} \\
& \equiv k_{c} .
\end{aligned}
$$

From the above analysis, we see that as " $k$ " passes through the value $k_{c}$, the system changes from unstable to stable state, that is, $k=k_{c}$ is a bifurcation point. 
In order to establish Hopf-bifurcation, we have to show that

$$
\left[\frac{d a}{d k}\right]_{k=k_{c}} \neq 0
$$

Differentiating (3.12) and (3.13) with respect to " $k$ " and solving for $d a / d k$, we get

$$
\frac{d a}{d k}=-\frac{2 A_{2} A_{3} m\left(T^{*}\right)^{m}}{\left(k+T^{*}\right)^{m}\left(E^{2}+F^{2}\right)} .
$$

Thus

$$
\left[\frac{d a}{d k}\right]_{k=k_{c}}=-\frac{2 A_{2}\left(A_{1} A_{2}-A_{3}\right)^{2}}{A_{3} m T^{*}\left(E^{2}+F^{2}\right)}<0 .
$$

Consequently, Hopf-bifurcation occurs near the equilibrium point $k=k_{c}$. Hence the theorem follows.

From Theorems 3.1 and 3.2, it follows that system (2.1) without delays, with $f(T)$ as in (3.11), will become unstable for $m>8$ and will undergo a Hopf-bifurcation at $k=k_{c}$ given by (3.19).

4. Bifurcation analysis in the presence of delays. We now study the stability of the interior equilibrium point $E^{*}$ in the presence of delays. The stability of $E^{*}$ can be determined by the sign of the real parts of the roots of (3.4). Let $\tau=\tau_{0}+\tau_{1}+\tau_{2}$ and let $\lambda=p+i q$. Substituting the value of $\lambda$ in (3.4) and separating real and imaginary parts, we get

$$
\begin{gathered}
p^{3}-3 p q^{2}+A_{1}\left(p^{2}-q^{2}\right)+A_{2} p+A_{3}+A_{4} e^{-p \tau} \cos q \tau=0, \\
3 p^{2} q-q^{3}+2 A_{1} p q+A_{2} q-A_{4} e^{-p \tau} \sin q \tau=0 .
\end{gathered}
$$

The necessary condition for a change of behaviour in the stability of $E^{*}$ is that one of the characteristic roots of system (3.2) is purely imaginary.

Let $\hat{\tau}$ be such that

$$
p(\hat{\tau})=0, \quad q(\hat{\tau})=\hat{q} .
$$

Then we can reduce (4.1) and (4.2) to

$$
\begin{aligned}
& A_{1} \hat{q}^{2}-A_{3}=A_{4} \cos \hat{q} \hat{\tau}, \\
& -\hat{q}^{3}+A_{2} \hat{q}=A_{4} \sin \hat{q} \hat{\tau} .
\end{aligned}
$$

Equations (4.4) and (4.5) together can be reduced to an equation in $\hat{q}^{2}$ of the form

$$
\Psi(\hat{q})^{2} \equiv\left(\hat{q}^{2}\right)^{3}+\left(A_{1}^{2}-2 A_{2}\right)\left(\hat{q}^{2}\right)^{2}+\left(A_{2}^{2}-2 A_{1} A_{3}\right) \hat{q}^{2}+\left(A_{3}^{2}-A_{4}^{2}\right) .
$$

We may rewrite (4.6) as a cubic equation in $\hat{q}^{2}$ as

$$
H(\eta) \equiv \eta^{3}+b_{1} \eta^{2}+b_{2} \eta+b_{3}=0,
$$


where

$$
b_{1}=A_{1}^{2}-2 A_{2}, \quad b_{2}=A_{2}^{2}-2 A_{1} A_{3}, \quad b_{3}=A_{3}^{2}-A_{4}^{2}, \quad \eta=\hat{q}^{2} .
$$

Now, $b_{1}=d_{1}^{2}+d_{2}^{2}+d_{3}^{2}$ is obviously positive.

Also, $b_{2}=A_{2}^{2}-2 A_{1} A_{3}=d_{1}^{2} d_{2}^{2}+d_{2}^{2} d_{3}^{2}+d_{3}^{2} d_{1}^{2}$ is also positive. Again,

$$
b_{3}=A_{3}^{2}-A_{4}^{2}=d_{1}^{2} d_{2}^{2} d_{3}^{2}-\left[f^{\prime}\left(T^{*}\right)\right]^{2} r_{2}^{2} r_{3}^{2} .
$$

LEMMA 4.1. (i) If $b_{3}<0$, then (4.7) has at least one positive root.

(ii) If $b_{3} \geq 0$ and $\Delta \equiv b_{1}^{2}-3 b_{2}<0$, then (4.7) has no positive roots.

(iii) If $b_{3} \geq 0$, then (4.7) has positive roots if and only if $\eta_{+}=(1 / 3)\left(-b_{1}+\sqrt{\Delta}\right)>0$ and $H\left(\eta_{+}\right) \leq 0$.

Proof. (i) We have $H(\eta)=\eta^{3}+b_{1} \eta^{2}+b_{2} \eta+b_{3}$. Obviously, $H(0)=b_{3}<0$ and

$$
\lim _{n \rightarrow \infty} H(\eta)=\infty
$$

Since $H(\eta)$ is continuous, there exists an $\eta_{0}$ with $0<\eta_{0}<\infty$ so that $H\left(\eta_{0}\right)=0$, that is, $\eta_{0}$ is a positive root of $H(\eta)=0$.

(ii) We have $H^{\prime}(\eta)=3 \eta^{2}+2 b_{1} \eta+b_{2}$. Since $b_{1}$ and $b_{2}$ are positive, then $H^{\prime}(\eta)>0$ for $\eta>0$. But the roots of $H^{\prime}(\eta)=0$ are

$$
\eta_{ \pm}=\frac{-2 b_{1} \pm \sqrt{4 b_{1}^{2}-12 b_{2}}}{6}=\frac{-b_{1} \pm \sqrt{\Delta}}{3} .
$$

Therefore, for $\Delta<0$, (4.7) must not have real roots, that is, $H^{\prime}(\eta) \neq 0$ for any real $\eta$. Consequently, $H^{\prime}(\eta)$, being a continuous function, is always positive, that is, $H(\eta)$ is monotone increasing and $H(0)=b_{3} \geq 0$. Therefore, (4.7) has no positive real roots.

(iii) The sufficiency part is obvious.

We assume that $\eta_{+} \leq 0$ or $\eta_{+}>0$ and $H\left(\eta_{+}\right)>0$. Now, if $\Delta \geq 0, H(\eta)$ has a local minimum at $\eta_{+}$. Thus $H(\eta)$ is increasing for $\eta>\eta_{+}$. If $\eta_{+} \leq 0$, then the equation $H(\eta)=0$ cannot have any positive real root as $H(0)=b_{3} \geq 0$.

If $\eta_{+}>0$ and $H\left(\eta_{+}\right)>0$, it follows that $H\left(\eta_{+}\right)<H\left(\eta_{-}\right)$as $H(\eta)$ has a local maxima at $\eta_{-}$and $H(\eta)$ is decreasing for $\eta_{-} \leq \eta$. Hence, from $H(0)=b_{3} \geq 0$, we may conclude that $H(\eta)=0$ has no positive roots.

We assume that (4.7) has positive roots. Let

$$
\hat{\tau}_{n}=\frac{1}{\hat{q}}\left[\arctan \frac{-\hat{q}^{3}+A_{2} \hat{q}}{A_{1} \hat{q}^{2}-A_{3}}+n \pi\right], \quad n=0,1,2, \ldots
$$

Then, $\lim _{n \rightarrow \infty} \hat{\tau}_{n}=\infty$. Suppose that $\tau_{0}=\min \left\{\hat{\tau}_{n}\right\}$ and the corresponding $\hat{q}$ is $\hat{q}_{0}$.

THEOREM 4.2. If system (2.1) without delay is asymptotically stable, then

(i) the stability of the system will remain unchanged for all $\tau \geq 0$ provided that $b_{3} \geq 0$ and $\Delta<0$;

(ii) the stability of the system will change for an infinite number of different values of $\tau$ given by (4.12) provided that $b_{3}<0$ or $b_{3} \geq 0, \eta_{+}>0$, and $H\left(\eta_{+}\right) \leq 0$; 
(iii) moreover,

$$
\frac{d}{d \tau} \operatorname{Re} \lambda\left(\tau_{0}\right)>0
$$

where $\tau_{0}$ is the minimum value of $\mathrm{\tau}$ for which stability change occurs, that is, the system exhibits Hopf-bifurcation provided that the conditions of (ii) are satisfied.

Proof. (i) For $\tau=0$, the system is asymptotically stable and consequently all the roots of (3.4) have negative real parts. For $\tau>0$, from Lemma 4.1(ii), we can say that (3.4) will have no roots with zero real part and there will be no change in the stability of the system.

(ii) If $b_{3}<0$ or $b_{3} \geq 0, \eta_{+}>0$, and $H\left(\eta_{+}\right) \leq 0$, then by Lemma 4.1(i) and (iii), equation (4.7) has positive roots and consequently (3.4) has roots with zero real parts, that is, a stability change will take place. The values of delays at which the stability change will occur are given by (4.12) and for $\tau \neq \hat{\tau}_{n}$, where $\hat{q}^{2}$ is a positive root of (4.7), equation (3.4) has no roots with zero real part. The value $\tau_{0}$ is the minimum value for which (3.4) has purely imaginary roots. It can also be proved that the system will be asymptotically stable for $\tau<\tau_{0}$.

(iii) Differentiating (3.4) with respect to $\tau$, we get

$$
\frac{d \lambda}{d \tau}=\frac{A_{4} \lambda e^{-\lambda \tau}}{3 \lambda^{2}+2 A_{1} \lambda+A_{2}-A_{4} \tau e^{-\lambda \tau}}
$$

Therefore,

$$
\frac{d}{d \tau}\left[\operatorname{Re} \lambda\left(\tau_{0}\right)\right]=\frac{q_{0}^{2}}{\Delta} H^{\prime}\left(\hat{q}_{0}^{2}\right)
$$

where

$$
\Delta=\left(A_{2}-3 \hat{q}_{0}^{2}-A_{4} \tau_{0} \cos \hat{q}_{0} \tau_{0}\right)^{2}+\left(2 A_{1} \hat{q}_{0}+A-4 \tau_{0} \sin \hat{q}_{0} \tau_{0}\right)^{2}
$$

and $H\left(\hat{q}_{0}^{2}\right)=3\left(\hat{q}_{0}^{2}\right)^{2}+2 b_{1} \hat{q}_{0}^{2}+b_{2}>0$ as $b_{1}, b_{2}>0$.

Hence, by Hopf-bifurcation theorem, we can say that the system will undergo a Hopfbifurcation at $\tau=\tau_{0}$.

From the above analysis, we see that if $b_{3}<0$, then the condition (ii) of Theorem 4.2 is satisfied and the system will exhibit Hopf-bifurcation at $\tau=\tau_{0}$. But $b_{3}<0$ implies that for small $k, m>1$, where $m$ is the Hill-coefficient, taking $f(T)$ as in (3.11).

5. Effects of castration and $T$ infusion. In this section, we first consider the hormonal changes of the system due to castration. After castration, the response rate for the production of $\mathrm{T}$ reduces to zero and hence the equilibrium points are

$$
\bar{R}=\frac{f(0)}{d_{1}}, \quad \bar{L}=\frac{r_{2} f(0)}{d_{1} d_{2}}, \quad \bar{T}=0
$$


Here

$$
\bar{L}=\frac{f(0)}{f\left(T^{*}\right)} L^{*}, \quad \bar{R}=\frac{f(0)}{f\left(T^{*}\right)} R^{*} .
$$

Considering $f(T)$ as in (3.11), we get

$$
\frac{f(0)}{f\left(T^{*}\right)}=\left[1+\frac{\left(T^{*}\right)^{m}}{k}\right]
$$

Therefore, $\bar{R}>R^{*}$ and $\bar{L}>L^{*}$ by a factor $1+\left(T^{*}\right)^{m} / k$. Now for small $k$, we know that the necessary condition for the pulsatile release prior to castration is $m>1$. Thus for small $k$, the postcastration LHRH and LH concentrations will be much higher than that of precastration concentrations. This result agrees with the experimental observations in many individuals [7].

Linearising system (2.1) about $(\bar{R}, \bar{L}, \bar{T})$ and considering $r_{3}=0$, we get the corresponding characteristic equation as

$$
\lambda^{3}+\left(d_{1}+d_{2}+d_{3}\right) \lambda^{2}+\left(d_{1} d_{2}+d_{2} d_{3}+d_{1} d_{3}\right) \lambda+d_{1} d_{2} d_{3}=0 .
$$

Proposition 5.1. System (2.1), after castration, is always asymptotically stable.

Proof. As the characteristic roots of (5.2) are all negative, namely, $-d_{1},-d_{2}$, and $-d_{3}$, the proof is obvious.

Next, we consider the effect of exogenous administration of T into the system. With this modification, the system becomes,

$$
\begin{aligned}
& \frac{d R}{d t}=f(T)-d_{1} R, \\
& \frac{d L}{d t}=r_{2} R\left(t-\tau_{1}\right)-d_{2} L, \\
& \frac{d T}{d t}=r_{3} L\left(t-\tau_{2}-\tau_{0}\right)-d_{3} T+\pi,
\end{aligned}
$$

where $\pi$ is the constant input of T. The new steady-state concentrations are

$$
R_{0}=\frac{f\left(T_{0}\right)}{d_{1}}, \quad L_{0}=\frac{r_{2} f\left(T_{0}\right)}{d_{1} d_{2}}, \quad T_{0}=\frac{r_{2} r_{3} f\left(T_{0}\right)}{d_{1} d_{2} d_{3}}+\frac{\pi}{d_{3}}
$$

The corresponding characteristic equation will be

$$
\lambda^{3}+C_{1} \lambda^{2}+C_{2} \lambda+C_{3}+C_{4} e^{-\lambda\left(\tau_{0}+\tau_{1}+\tau_{2}\right)}=0
$$

where

$$
\begin{gathered}
C_{1}=d_{1}+d_{2}+d_{3}, \quad C_{2}=d_{1} d_{2}+d_{2} d_{3}+d_{3} d_{1}, \\
C_{3}=d_{1} d_{2} d_{3}, \quad C_{4}=-f^{\prime}\left(T_{0}\right) r_{2} r_{3} .
\end{gathered}
$$


Taking $f(T)=A /\left(k+T^{m}\right)$ and using Theorem 4.2(i), we conclude that there will be no stability change if

$$
\frac{d_{1} d_{2} d_{3} T_{0}}{r_{2} r_{3}} \geq \frac{A m T_{0}^{m}}{\left(k+T_{0}^{m}\right)^{2}},
$$

where $T_{0}$ is the root of the equation

$$
T^{m+1} d_{1} d_{2} d_{3}+T^{m} \pi d_{2} d_{3}+k d_{1} d_{2} d_{3} T+k \pi d_{2} d_{3}-A r_{2} r_{3}=0
$$

From this, we may conclude that proper infusion of $\mathrm{T}$ at a constant rate may cause the suppression of the pulsatile or episodic release.

6. Discussion. In this paper, we have considered a complete LHRH-LH-T system model based on certain qualitative experimental observations. The model partly shows its utility in predicting several known results but mainly provides a concrete framework in which one's understanding of the physicochemical system is made explicit. The most important feature of the model is its "built-in" clock-like biochemical oscillator. Some other models of the reproductive system incorporate an external driving function to mimic the oscillations. Such types of models, we feel, will be of limited value in understanding the system.

In Section 3, we have performed the stability analysis of the system in the absence of delays and found that unstability will occur for $m>8$ approximately, where " $m$ " is the Hill coefficient of the feedback function. It has also been shown that the unstability leads to the oscillation of the system at the bifurcation value $k=k_{c}$.

In Section 4, we have considered the delay effects, namely, the transportation delays of LHRH and LH into the bloodstream and synthesis delay of T. The stability analysis, in this case, reveals that the system will be unstable for $m>1$ approximately. From this analysis, we conclude that, in the absence of delays, the system will be unstable for $m>$ 8 which is unnaturally high [11], whereas the same condition reduces to $m>1$ when delays are considered. Hence, the introduction of different delays makes the system much more realistic as the conclusion in this case conforms with the experimental observations more closely.

We have also shown that periodic fluctuation of $\mathrm{T}$ level in the bloodstream occurs for an infinite number of values of the parameter $\tau$ which represents the sum of the system delays. The transportation delays of different hormones in the bloodstream and the synthesis delay of $\mathrm{T}$ (that is the interval between testis stimulation and T release) vary among individuals. But the phenomenon of periodic fluctuations of blood levels of $\mathrm{T}$ is present in all individuals though the periods may be different. This experimental observation also supports our findings, as the sum of transportation and synthesis delays for any particular individual may be taken as one of the infinite number of values we have found for which oscillation of $\mathrm{T}$ level in the blood takes place and hence establishes the phenomenon of periodic oscillation of $\mathrm{T}$ for all individuals.

Lastly, we have studied the effects of castration and T infusion. Our findings in the first case showed that for small values of " $k$," the serum concentration of LHRH and LH will be much higher than normal in castrated animals. This result is in accordance with the experimental observations of Dierschke et al. [7]. Regarding hormone therapy, 
our analysis revealed that the pulsatile release of different hormones into the bloodstream can be suppressed by infusion of $\mathrm{T}$ at a specified constant rate-a phenomenon observed experimentally by Bolt [2].

ACKNOWLEDgment. The authors are grateful to Prof. C. G. Chakrabarti, SN. Bose Professor, Department of Applied Mathematics, University of Calcutta, for his continuous help and guidance throughout the preparation of the paper.

\section{REFERENCES}

[1] A. Arimura, Hypothalamic gonadotropin-releasing hormone and reproduction, International Review of Physiology, Reproductive Physiology-II (R. O. Greep, ed.), vol. 13, Un. Park Press, Baltimore, 1977, pp. 1-21.

[2] P. J. Bolt, Changes in the concentration of luteinizing hormone in plasma of rams following administration of oestradiol, progesterone or testosterone, J. Reprod. Fert. 24 (1971), 435-438.

[3] K. Brown-Grant, Control of gonadotropin secretion, Subcellular Mechanisms in Reproductive Neuroendocrinology (F. Naftolin, K. J. Ryan, and J. Davis, eds.), Elsevier Scientific Publishing, Amsterdam, 1976.

[4] _ Physiological aspects of the steroid hormone-gonadotropin interrelationship, International Review of Physiology, Reproductive Endocrinology II, vol. 13, 1977, pp. 5783.

[5] P. W. Carmel, S. Araki, and M. Ferin, Pituitary stalk portal blood collection in rhesus monkeys: evidence for pulsatile release of gonadotropin-releasing hormone, Endocrinology 99 (1976), 243-248.

[6] M. Courot, Hormonal regulation of male reproduction (with reference to infertility in man), Andralogia 8 (1976), 187-193.

[7] D. J. Dierschke, F. J. Karsh, R. F. Weick, G. Weiss, J. Hotchkiss, and E. Knobil, Hypothalamicpituitary regulation of puberty: feedback control of gonadotropin secretion in the rhesus monkey, Control of Onset of Puberty (M. M. Grumbach, A. D. Grave, and F. E. Mayer, eds.), Wiley, New York, 1974.

[8] G. H. Hardy, J. E. Littlewood, and G. Pólya, Inequalities, Cambridge University Press, Cambridge, 1952.

[9] C. B. Katongole, F. Naftolin, and R. V. Short, Relationship between blood levels of luteinizing hormone and testosterone in bulls and their effects on sexual stimulation, J. Endocr. 50 (1971), 457-466.

[10] W. H. Moger and D. T. Armstrong, Changes in serum testosterone levels following acute LH treatment in immature and mature rats, Biol. Reprod. 11 (1974), 1-6.

[11] J. D. Murray, Mathematical Biology, Biomathematics, vol. 19, Springer-Verlag, Berlin, 1993.

[12] H. R. Nankin and P. Troen, Repetitive luteinizing hormone elevations in serum of normal men, J. Clin. Endocrin. Metab. 35 (1971), 931-937.

[13] J. Pelletier, Elevation du taux de LH dans le plasma sanguin du belin cepres castration, Ann. Biol. Anim. Biophys. 8 (1968), 313-315 (French).

[14] _ Mode of action of testosterone proprianate on the secretion and release of luteinizing hormone (LH) in the castrated ram, Acta. Endocr. 63 (1970), 290-308.

Banibrata Mukhopadhyay: Department of Applied Mathematics, University of Calcutta, Calcutta 700 009, India

E-mail address: banibrat001@yahoo.co. in

Rakhi Bhattacharyya: Department of Applied Mathematics, University of Calcutta, Calcutta 700 009, India

E-mail address: rakhi_bhattach@yahoo.co.in 


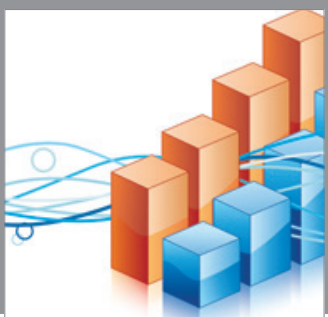

Advances in

Operations Research

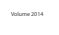

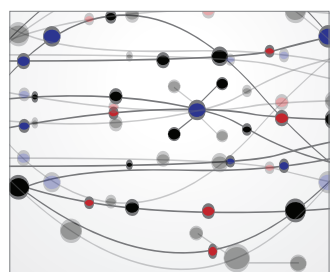

\section{The Scientific} World Journal
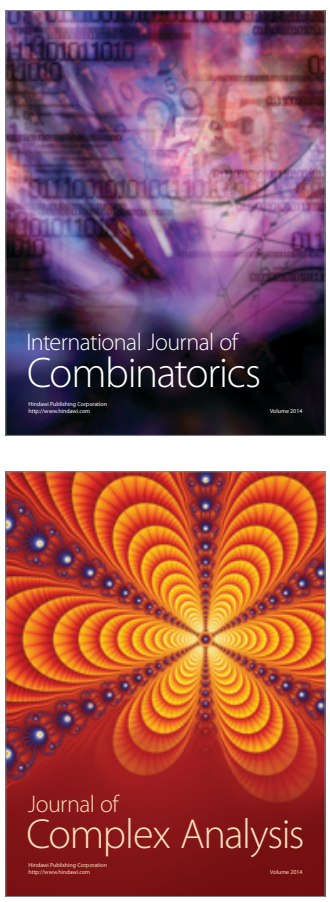

International Journal of

Mathematics and

Mathematical

Sciences
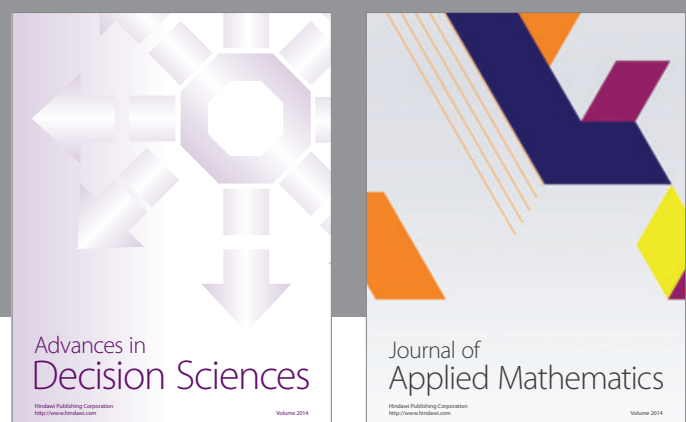

Journal of

Applied Mathematics
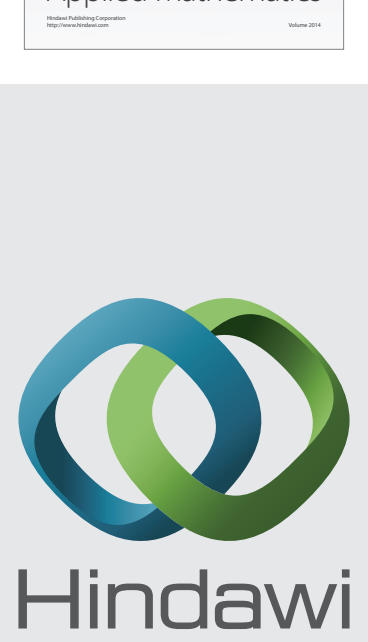

Submit your manuscripts at http://www.hindawi.com
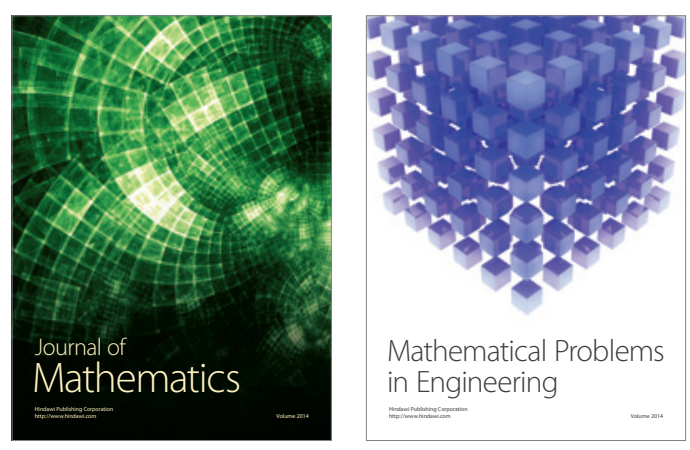

Mathematical Problems in Engineering
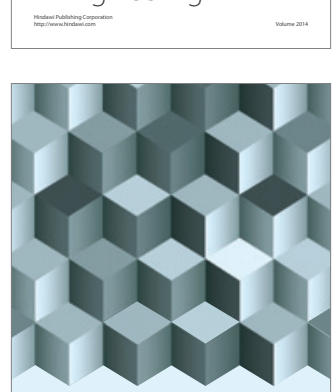

Journal of

Function Spaces
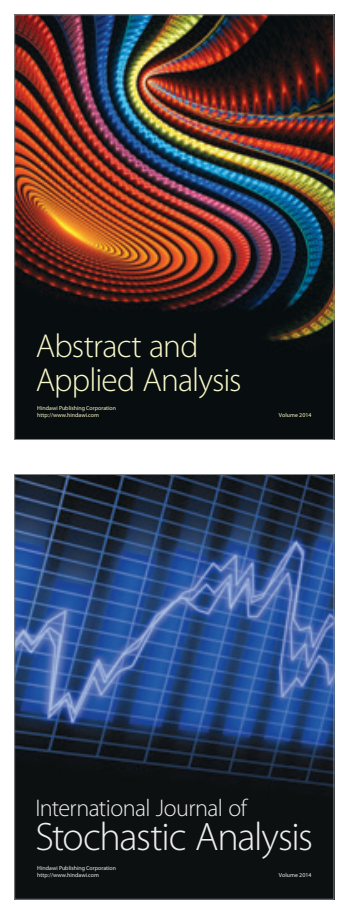

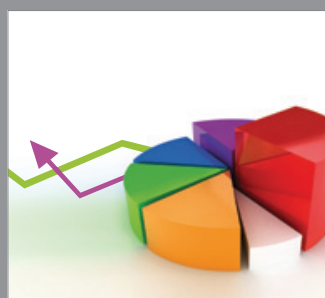

ournal of

Probability and Statistics

Promensencen
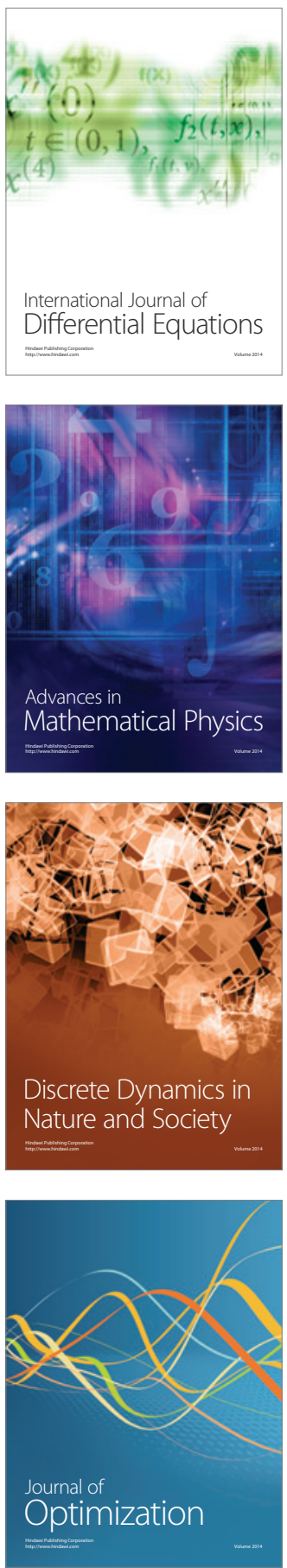